\title{
Environmental oxidative aging of iron oxide nanoparticles
}

Lara K. Bogart, Cristina Blanco-Andujar, and Quentin A. Pankhurst

Citation: Appl. Phys. Lett. 113, 133701 (2018); doi: 10.1063/1.5050217

View online: https://doi.org/10.1063/1.5050217

View Table of Contents: http://aip.scitation.org/toc/apl/113/13

Published by the American Institute of Physics

\section{Articles you may be interested in}

Flexoelectricity in antiferroelectrics

Applied Physics Letters 113, 132903 (2018); 10.1063/1.5044724

Optical fiber-driven low energy electron gun for ultrafast streak diffraction

Applied Physics Letters 113, 133502 (2018); 10.1063/1.5039737

Yttrium zinc tin oxide high voltage thin film transistors

Applied Physics Letters 113, 132101 (2018); 10.1063/1.5048992

Miniature all-fibre microflown directional acoustic sensor based on crossed self-heated micro-Co ${ }^{2+}$-doped optical fibre Bragg gratings

Applied Physics Letters 113, 134102 (2018); 10.1063/1.5043519

Fast spatial scanning of 3D ultrasound fields via thermography

Applied Physics Letters 113, 133503 (2018); 10.1063/1.5046834

Breakdown mechanism in $1 \mathrm{kA} / \mathrm{cm}^{2}$ and $960 \mathrm{~V}$ E-mode $\beta-\mathrm{Ga}_{2} \mathrm{O}_{3}$ vertical transistors

Applied Physics Letters 113, 122103 (2018); 10.1063/1.5038105

\section{Conference Proceedings}




\title{
Environmental oxidative aging of iron oxide nanoparticles
}

\author{
Lara K. Bogart, ${ }^{1}$ Cristina Blanco-Andujar, ${ }^{1,2}$ and Quentin A. Pankhurst ${ }^{1, a)}$ \\ ${ }^{1}$ UCL Healthcare Biomagnetics Laboratory, University College London, 21 Albemarle Street, \\ London WIS 4BS, United Kingdom \\ ${ }^{2}$ MediSieve Limited, Translation and Innovation Hub, 80 Wood Lane, London W12 OBZ, United Kingdom
}

(Received 28 July 2018; accepted 4 September 2018; published online 25 September 2018)

\begin{abstract}
The environmental aging of functional iron oxide nanomaterials, especially those used in biomedical applications, may have significant consequences for both their mechanism of action and their safety profile. However, to date, there has been no way to systematically measure this property, and the problem has been largely overlooked. We show here that a recently proposed "center of gravity" method for determining the composition of magnetite $\left(\mathrm{Fe}_{3} \mathrm{O}_{4}\right)$ and maghemite $\left(\gamma\right.$ - $\left.\mathrm{Fe}_{2} \mathrm{O}_{3}\right)$ mixtures via ${ }^{57} \mathrm{Fe}$ Mössbauer spectroscopy can be applied to the matter. We show that a long-established diffusion model can be used to characterize the oxidative aging process, yielding a parametric (and therefore predictive) description of the magnetite-to-maghemite oxidation processes occurring within the nanoparticles. Published by AIP Publishing. https://doi.org/10.1063/1.5050217
\end{abstract}

Functional iron oxide nanomaterials are widely used in biomedical applications, with currently approved agents being used in magnetic resonance imaging, sentinel node detection, iron replacement therapy, and magnetic thermoablation and with many other prospects such as targeted drug delivery being actively explored. ${ }^{1,2}$ These nanomaterials almost invariably comprise magnetite $\left(\mathrm{Fe}_{3} \mathrm{O}_{4}\right)$ and/or its oxidation product, maghemite $\left(\gamma-\mathrm{Fe}_{2} \mathrm{O}_{3}\right)$, both of which have high room-temperature magnetizations. ${ }^{3}$ At an atomic level, these two phases are quite different, with magnetite being a mixed-valent oxide containing both ferrous $\left(\mathrm{Fe}^{2+}\right)$ and ferric $\left(\mathrm{Fe}^{3+}\right)$ ions in a 1:2 molar ratio, whereas all of the iron in maghemite is in the $\mathrm{Fe}^{3+}$ state. However, at a macroscopic level, the nanoscale form leads to experimental effects, such as the broadening of X-ray diffraction lines, which render the two phases virtually indistinguishable. Recognition of this difficulty has led to the adoption in the field of an unofficial convention, whereby a product of unknown composition is commonly referred to as "magnetite/maghemite."

At the same time, for biomedical applications, there is increasing recognition of the importance of the redox-active aspects of these iron oxides, which depend implicitly on the molar ratio of ferrous and ferric ions, $x=\mathrm{Fe}^{2+} / \mathrm{Fe}^{3+}$. This applies both to prospectively beneficial mechanisms of action, such as in the recently proposed fields of macrophage phenotype activation ${ }^{4,5}$ and enzyme mimetics, ${ }^{6}$ and to potential safety concerns related to the production of reactive oxygen species via the catalytic Haber-Weiss and Fenton chemistry pathways. ${ }^{7,8}$ In this context, there is a clear need for a reliable method of measuring $x$ in the nanoparticulate state. Furthermore, it is important to be able to characterize the time-evolution of $x$ under standard environmental (e.g., storage) conditions, as this may significantly affect both the material's in vivo mechanism of action and its safety profile.

To this end, we apply here the "center of gravity" (COG) method $^{9}$ that allows for the $\mathrm{Fe}^{2+} / \mathrm{Fe}^{3+}$ molar ratio in magnetite/maghemite nanoparticles to be non-destructively

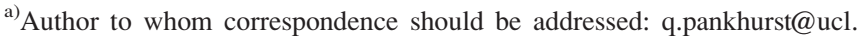
ac.uk
}

determined $v i{ }^{57} \mathrm{Fe}$ Mössbauer spectroscopy, and we monitor thereby the oxidative aging process in relation to the timedependent transformation of $\mathrm{Fe}^{2+}$ to $\mathrm{Fe}^{3+}$ ions. Mössbauer spectroscopy has been used previously to explore the aging of iron oxide nanoparticles, ${ }^{10}$ but the use here of the COG method allows for a more systematic and quantitative analysis to be performed.

For illustrative purposes, two similar but different magnetite/maghemite nanoparticle systems were studied. Both were bare (i.e., uncoated) single-core nanoparticle systems. ${ }^{11}$ Both had log-normal core size distributions (as evidenced by transmission electron microscopy), with comparable median diameters of ca. $14.9 \mathrm{~nm}$ and $16.8 \mathrm{~nm}$ and log-normal standard deviations of ca. 0.13 and 0.19 , respectively. The structural and magnetic properties of both samples, as measured by X-ray diffraction and SQUID magnetometry (the latter including hysteresis data at $5 \mathrm{~K}$ and $300 \mathrm{~K}$ and variable temperature remanent magnetization data), were almost indistinguishable. As such they were, ostensibly at least, very similar materials.

The samples were, however, the products of quite different synthesis routes. Sample $\mathrm{S} 1$ was produced via the $\mathrm{Na}_{2} \mathrm{CO}_{3}$-reduction co-precipitation method under airsensitive conditions and conventional heating, ${ }^{12}$ whereas sample $\mathrm{S} 2$ was produced via $\mathrm{Na}_{2} \mathrm{CO}_{3}$-reduction co-precipitation in air, with microwave-assisted heating. ${ }^{13}$ Both were freeze-dried immediately after synthesis to produce a friable powder, which was then subsequently allowed to age in air. On the basis of their different synthesis pathways, it was anticipated that S1 would be more susceptible to oxidative aging than S2, having been produced under reduced-oxygen conditions.

To test this hypothesis, the $\mathrm{Fe}^{2+} / \mathrm{Fe}^{3+}$ molar ratios of both samples were serially measured over more than three years using the Mössbauer COG method, as introduced by da Costa et al. ${ }^{14}$ and as elucidated by Fock et al. ${ }^{9,15}$ According to the COG method, a single parameter $\left(\bar{\delta}_{R T}\right)$ the "center of gravity" or area weighted mean isomer shift at room temperature, $T=295 \pm 5 \mathrm{~K}$-is extracted by curvefitting a spectrum using a partly constrained ${ }^{16}$ superposition 


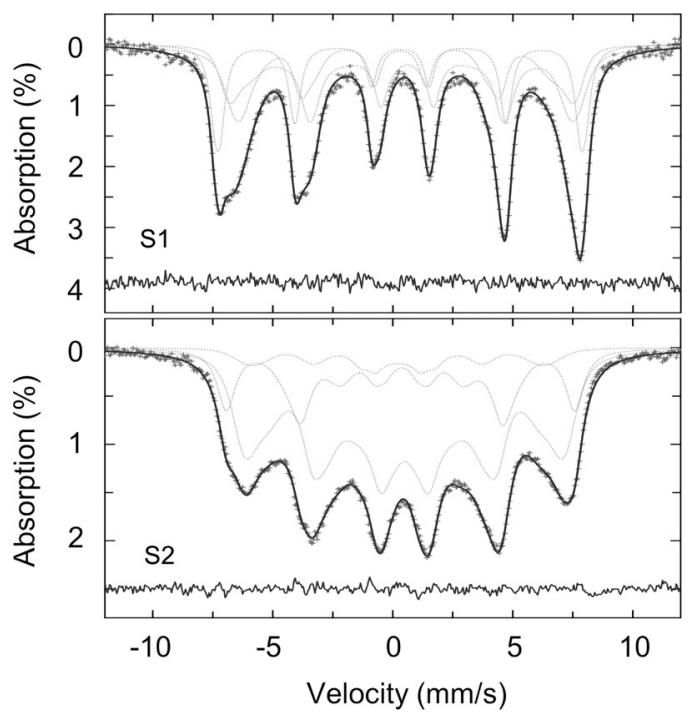

FIG. 1. Room temperature ${ }^{57} \mathrm{Fe}$ Mössbauer spectra of magnetite/maghemite samples S1 and S2, recorded 1 day after synthesis. The solid lines indicate the results of least-squares fitting of the spectra using Voigtian line profiles (Gaussian distributions of Lorentzian lines).

of Lorentzian singlets, doublets, and sextets. The $\bar{\delta}_{R T}$ parameter correlates with the number of Fe atoms in the magnetite environment: $\alpha=\mathrm{Fe}_{\text {magnetite }} / \mathrm{Fe}_{\text {total }}=\left(\bar{\delta}_{R T}-\delta_{\mathrm{o}}\right) / m$, where $\delta_{\mathrm{o}}=0.3206 \pm 0.0022 \mathrm{~mm} / \mathrm{s}$ and $m=0.2135 \pm 0.0076 \mathrm{~mm} / \mathrm{s}^{9}$ This in turn leads to the molar ratio, as $x=\alpha /(3-\alpha)$.

Room temperature Mössbauer spectra of the as-made S1 and $\mathrm{S} 2$ samples, recorded on day 1, are shown in Fig. 1. It is evident that the absorption lines in the S1 spectrum are narrower than those in the S2 spectrum, which may indicate that the microwave-mediated accelerated nucleation and growth route used for sample S2 leads to a more disordered and/or less crystalline local structure.

The measured $\bar{\delta}_{R T}$ and derived $x$ values over the entire three-year course of experiments are shown in Fig. 2. In both $\mathrm{S} 1$ and $\mathrm{S} 2$, it is clear that there is a steady process of oxidative aging, with both samples starting with ca. 20 at. $\%$ of the iron in the $\mathrm{Fe}^{2+}$ state and both reaching much lower values of ca. 2 at. $\%$ after three years.

To analyze the oxidative aging process, it is useful to review the mechanics and mathematics of how magnetite

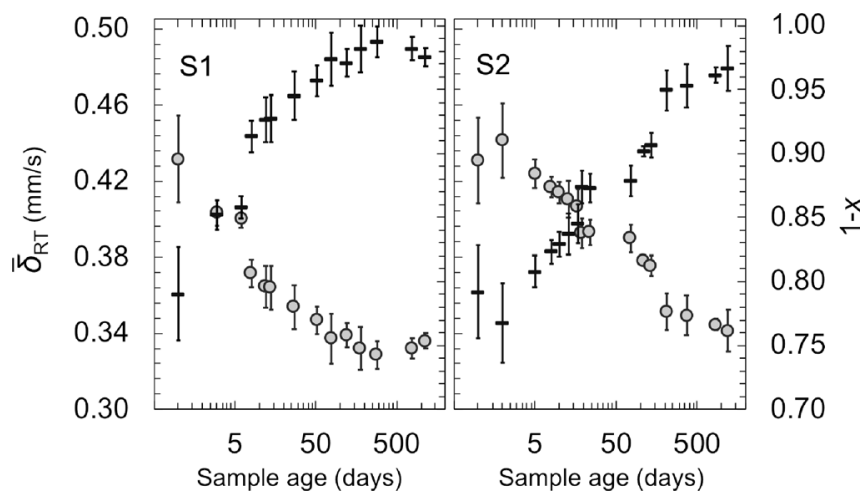

FIG. 2. Measured $\bar{\delta}_{R T}$ area weighted mean isomer shifts (circles) and derived $x=\mathrm{Fe}^{2+} / \mathrm{Fe}^{3+}$ molar ratios (bars) obtained by analyzing the ${ }^{57} \mathrm{Fe}$ Mössbauer spectra of samples S1 and S2 using the COG method. The spectra were recorded serially over more than 3 years, during which time the powder samples were exposed to environmental aging in air. oxidizes to maghemite. Contrary to common expectation, the oxidation of magnetite nanoparticles does not occur by oxygen diffusing into the particle but rather by the diffusion of iron ions out of the particle. ${ }^{17}$ That is, the particles oxidize "from the inside out." The process has two steps. First, oxygen is adsorbed to the surface of the nanoparticle where it becomes ionized by those electrons that are released by the oxidation of $\mathrm{Fe}^{2+}$ to $\mathrm{Fe}^{3+}$, thereby preserving the charge neutrality of the material. ${ }^{17}$ Second, the surface oxidation results in a concentration gradient, ${ }^{18}$ which leads to the further diffusion of iron ions to the surface.

It should be noted that it is not entirely certain which of the iron species diffuses from the core to the surface or whether it may be both. Plausible mechanisms exist for both (a) $\mathrm{Fe}^{2+}$ ions being drawn out from the core by the $\mathrm{Fe}^{2+}$ concentration gradient set up by the surface oxidation process, and (b) internal $\mathrm{Fe}^{2+}$ ions being oxidized in situ through encounters with mobile electrons, and the resultant $\mathrm{Fe}^{3+}$ ions diffusing away from the site to maintain the charge neutrality of the structure. ${ }^{19}$ In the analysis to follow, we will describe the oxidation process in terms of the increasing concentration of $\mathrm{Fe}^{3+}$ ions in the material, $\left[\mathrm{Fe}^{3+}\right]_{t}$, which varies from $66.7 \%$ in magnetite to $100 \%$ in maghemite, but we do so only as a matter of convenience.

Turning to the mathematical models of diffusion, several authors have reported on the kinetics of "low temperature" (meaning ca. $220^{\circ} \mathrm{C}$ ) oxidation in micron-sized magnetite particles. ${ }^{19,20}$ In these works, the diffusion coefficient was obtained by solving Fick's second equation: a partial differential equation that describes diffusion due to a time-varying concentration of the diffusing substance. For simplicity, it is usual to assume spherical particles for which oxidation is both isotropic and occurs radially via the diffusion of ions from the core to the surface. The non-steady state solution to this, provided by Crank, ${ }^{21}$ is expressed in series form as

$$
\frac{M_{t}}{M_{\infty}}=1-\frac{6}{\pi^{2}} \sum_{n=1}^{\infty} \frac{1}{n^{2}} \exp \left(-D n^{2} \pi^{2} t / r^{2}\right),
$$

where $M_{t}$ is the quantity of Fe that has diffused out of a sphere of radius $r$ in a time $t, M_{\infty}$ is the corresponding quantity at $t=\infty$, and $D$ is the diffusion coefficient.

We apply this to the COG data by noting that

$$
\left[\mathrm{Fe}^{3+}\right]=\frac{1}{1+x}=1-\frac{\alpha}{3}
$$

and by defining the experimentally determined initial and terminal $\mathrm{Fe}^{3+}$ concentrations as $\left[\mathrm{Fe}^{3+}\right]_{0}$ and $\left[\mathrm{Fe}^{3+}\right]_{\infty}$, so that

$$
\frac{M_{t}}{M_{\infty}}=\frac{\left[\mathrm{Fe}^{3+}\right]_{t}-\left[\mathrm{Fe}^{3+}\right]_{0}}{\left[\mathrm{Fe}^{3+}\right]_{\infty}-\left[\mathrm{Fe}^{3+}\right]_{0}} .
$$

This expression then allows for a straightforward numerical evaluation $^{22}$ of Eq. (1) to be obtained, which can be compared to the experimental data in Eq. (3), and in turn can provide an estimation of sample-specific diffusion coefficients, $D$.

Applying this analysis to samples $\mathrm{S} 1$ and $\mathrm{S} 2$ results in the data presented in Fig. 3 and Table I. On inspection, it is 


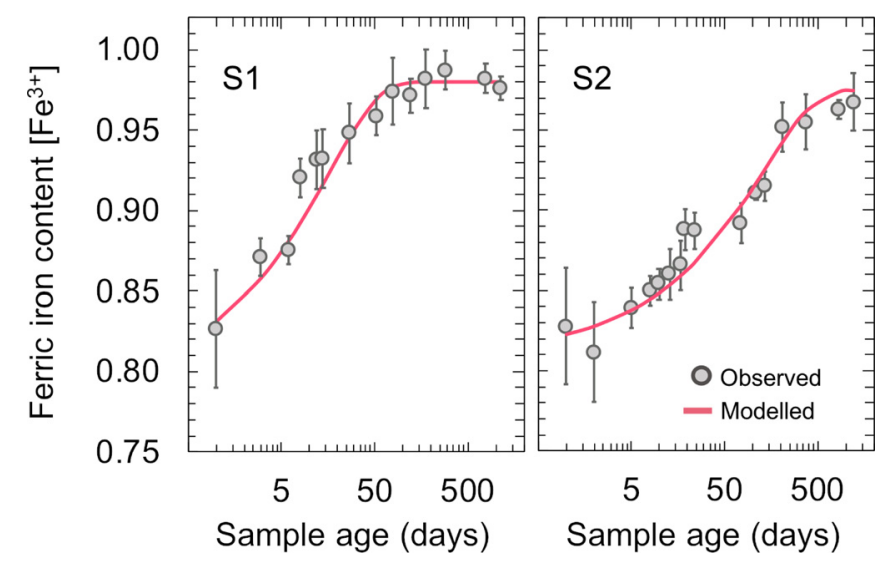

FIG. 3. Derived $\left[\mathrm{Fe}^{3+}\right]_{t}$ concentration data obtained by analyzing the ${ }^{57} \mathrm{Fe}$ Mössbauer spectra of samples S1 and S2 using the COG method and applying Eq. (3), indicating that the oxidative aging process is quite different in the two samples. The solid lines are simulations based on Eqs. (1) and (3), using the parameters listed in Table I.

apparent that the fits are very good and that they accurately reflect the features in the data, notably the approach to saturation in $\mathrm{S} 1$, and the absence thereof in $\mathrm{S} 2$. Both samples had initial $\mathrm{Fe}^{3+}$ concentrations of ca. $80 \%$, indicating that neither was a "pure" magnetite at the outset, and both samples had terminal $\mathrm{Fe}^{3+}$ concentrations of ca. $98 \%$, indicating that some persistent residual $\mathrm{Fe}^{2+}$ ions remain, presumably in the particle cores.

The most striking difference between samples S1 and S2 lies in their diffusion coefficients, which differ by an order of magnitude. $\mathrm{S} 1$ has the faster diffusion (larger $D$ ), as evidenced by its relatively rapid approach to $\left[\mathrm{Fe}^{3+}\right]_{\infty}$ saturation. Thus, for example, $\mathrm{S} 1$ loses half of its initial $\mathrm{Fe}^{2+}$ content and reaches an $\left[\mathrm{Fe}^{3+}\right]_{t}$ level of $90 \%$ after ca. 10 days, while it takes $\mathrm{S} 2$ ca. 70 days to reach the same level. This is consistent with our initial expectation that since sample S1 was prepared under anaerobic conditions, it would be more susceptible to oxidative aging than S2. That said, the Mössbauer data of Fig. 1 showed that S2 was significantly less crystalline (as evidenced by the broader absorption lines) than S1, and reduced crystallinity has in the past been reported as a factor in promoting oxidation rates. ${ }^{17}$ As such, it appears that there may be competing factors at work in these two samples, determining the overall diffusion rates.

It is notable, and somewhat surprising, that in both cases, the diffusivities are rather low. Sidhu et al. ${ }^{19}$ reported that for the micron-sized magnetite particles that they studied, the diffusion coefficients followed an Arrhenius type law

$$
D=D_{\mathrm{o}} \exp (-E / R T)
$$

TABLE I. Parameters used to describe the oxidative aging process in magnetite/maghemite samples $\mathrm{S} 1$ and $\mathrm{S} 2$ : the median radius of the particles $r$; the initial and terminal $\mathrm{Fe}^{3+}$ concentrations $\left[\mathrm{Fe}^{3+}\right]_{0}$ and $\left[\mathrm{Fe}^{3+}\right]_{\infty}$; and the diffusion coefficients $D$. The numbers in brackets represent estimated uncertainties in the last digits of the parameters.

\begin{tabular}{lcccc}
\hline \hline Sample & $r(\mathrm{~nm})$ & {$\left[\mathrm{Fe}^{3+}\right]_{0}(\%)$} & {$\left[\mathrm{Fe}^{3+}\right]_{\infty}(\%)$} & $D\left(\mathrm{~m}^{2} / \mathrm{s}\right)$ \\
\hline S1 & $7.5(1)$ & $79.0(5)$ & $98.0(5)$ & $3.0(5) \times 10^{-24}$ \\
S2 & $8.4(1)$ & $81.0(5)$ & $97.5(5)$ & $4.5(5) \times 10^{-25}$ \\
\hline \hline
\end{tabular}

where $R$ is the gas constant, and $D_{\mathrm{o}} \approx 3.16 \times 10^{-9} \mathrm{~m}^{2} / \mathrm{s}$ and $E \approx 81 \mathrm{~kJ} / \mathrm{mol}$ are experimentally determined constants. Applying this equation to $T=295 \mathrm{~K}$, one obtains $D \approx 1.3$ $\times 10^{-23} \mathrm{~m}^{2} / \mathrm{s}$, which is $4 \times$ larger (i.e., faster) than in $\mathrm{S} 1$ and $29 \times$ larger than in S2. It is not immediately clear why it should be the case that the diffusivity of the iron ions in these materials should be so low.

To explore this further, it would be interesting to undertake a more comprehensive study of the oxidative aging of a broader range of magnetite/maghemite nanomaterials. In particular, one might anticipate that factors such as particle size, morphology, crystallinity, and impurity levels may all play a role in determining the diffusion properties.

It is also worth noting that the analysis as applied here has been intentionally simplistic, for example, by ascribing a single mean particle size to the sample (viz. the median size from the measured log normal distribution), rather than taking account of its polydispersity. It is possible that a more detailed study, allowing for possible variations in diffusivity as a function of particle size, might provide further insight into the underlying processes.

In any case, it is clear that the Mössbauer-based COG method is a useful tool in the study of environmental oxidative aging in magnetite/maghemite nanomaterials. It is an inherently non-destructive measurement modality, which allows longitudinal studies to be performed over days, weeks, months, and years. It provides substantive data on the relative concentrations of $\mathrm{Fe}^{2+}$ and $\mathrm{Fe}^{3+}$ ions in a sample and lends itself to modelling and the extraction of characteristic parameters such as the diffusion coefficient, $D$. This then allows quantitative comparisons to be made between samples, which may support future research initiatives, such as efforts to prolong the shelf-life of magnetite, ${ }^{2,24}$ or the synthesis of magnetite nanoparticles with oxidation-resistant coating layers. $^{25,26}$

See supplementary material for further details on the structural and magnetic properties of the samples and the Mössbauer experiments and fits.

This work was supported in part by the European Commission Seventh Framework Programme through the NanoMag project, under Grant No. 604448.

${ }^{1}$ Q. A. Pankhurst, J. Connolly, S. K. Jones, and J. Dobson, J. Phys. D: Appl. Phys. 36, R167 (2003).

${ }^{2}$ Q. A. Pankhurst, N. K. T. Thanh, S. K. Jones, and J. Dobson, J. Phys. D: Appl. Phys. 42, 224001 (2009).

${ }^{3}$ B. D. Cullity and C. D. Graham, Introduction to Magnetic Materials (John Wiley \& Sons, Hoboken, 2011).

${ }^{4}$ S. Zanganeh, G. Hutter, R. Spitler, O. Lenkov, M. Mahmoudi, A. Shaw, J. S. Pajarinen, H. Nejadnik, S. Goodman, M. Moseley et al., Nat. Nanotechnol. 11, 986 (2016).

${ }^{5}$ J. M. Rojas, L. Sanz-Ortega, V. Mulens-Arias, L. Gutierrez, S. PerezYague, and D. F. Barber, Nanomed.-Nanotechnol. Biol. Med. 12, 1127 (2016).

${ }^{6}$ L. Gao, K. Fan, and X. Yan, Theranostics 7, 3207 (2017).

${ }^{7}$ K. Rusevova, F.-D. Kopinke, and A. Georgi, J. Hazard. Mater. 241, 433 (2012).

${ }^{8}$ E.-J. Park, H. N. Umh, D.-H. Choi, M. H. Cho, W. Choi, S.-W. Kim, Y. Kim, and J.-H. Kim, Arch. Toxicol. 88, 1607 (2014).

${ }^{9}$ J. Fock, L. K. Bogart, D. González-Alonso, J. I. Espeso, M. F. Hansen, M. Varón, C. Frandsen, and Q. A. Pankhurst, J. Phys. D: Appl. Phys. 50, 265005 (2017). 
${ }^{10}$ C. Rümenapp, F. E. Wagner, and B. Gleich, J. Magn. Magn. Mater. 380, 241 (2015).

${ }^{11}$ J. Wells, O. Kazakova, O. Posth, U. Steinhoff, S. Petronis, L. K. Bogart, P. Southern, Q. A. Pankhurst, and C. Johansson, J. Phys. D: Appl. Phys. 50, 383003 (2017).

${ }^{12}$ C. Blanco-Andujar, D. Ortega, Q. A. Pankhurst, and N. T. K. Thanh, J. Mater. Chem. 22, 12498 (2012).

${ }^{13}$ C. Blanco-Andujar, D. Ortega, P. Southern, Q. A. Pankhurst, and N. T. K. Thanh, Nanoscale 7, 1768 (2015).

${ }^{14}$ G. M. da Costa, C. Blanco-Andujar, E. De Grave, and Q. A. Pankhurst, J. Phys. Chem. B 118, 11738 (2014).

${ }^{15}$ J. Fock, L. K. Bogart, O. Posth, M. F. Hansen, Q. A. Pankhurst, and C. Frandsen, Hyperfine Interact. 237, 23 (2016).

${ }^{16}$ Doublet lines should be of equal area; in sextets the area ratios of the outermost:middle:innermost pairs should be $3: 2: 1$.

${ }^{17}$ U. Colombo, G. Sironi, F. Gazzarri, G. Fagheraz, and G. Lanzavec, Nature 202, 175 (1964).

${ }^{18}$ Maghemite is less dense than magnetite but has the same underlying structure; hence the "concentration gradient" here can be thought of as the concentration of iron ions in the oxygen matrix, which is higher in the more magnetite-like particle core than in the more maghemite-like surface shell.

${ }^{19}$ P. S. Sidhu, R. J. Gilkes, and A. M. Posner, J. Inorg. Nucl. Chem. 39, 1953 (1977).

${ }^{20}$ K. J. Gallagher, W. Feitknecht, and U. Mannweiler, Nature 217, 1118 (1968).

${ }^{21}$ J. Crank, The Mathematics of Diffusion (Oxford University Press, New York, 1975), Chap. 6.3.

${ }^{22}$ The summation was performed with $\mathrm{n}=50$ terms.

${ }^{23}$ M. Widdrat, M. Kumari, E. Tompa, M. Posfai, A. M. Hirt, and D. Faivre, ChemPlusChem 79, 1225 (2014).

${ }^{24}$ A. Nistler, C. Hartmann, C. Ruemenapp, M. Opel, B. Gleich, N. P. Ivleva, R. Niessner, and M. Seidel, J. Magn. Magn. Mater. 442, 497 (2017).

${ }^{25}$ K. Cendrowski, P. Sikora, B. Zielinska, E. Horszczaruk, and E. Mijowska, Appl. Surf. Sci. 407, 391 (2017).

${ }^{26}$ S. W. da Silva, L. R. Guilherme, A. C. de Oliveira, V. K. Garg, P. A. Matos Rodrigues, J. A. Huamani Coaquira, Q. d. S. Ferreira, G. H. Ferreira de Melo, A. Lengyel, R. Szalay et al., J. Radioanal. Nucl. Chem. 312, 111 (2017). 